\title{
Evonik baut Anlage zur Herstellung funktionalisierter Polybutadiene
}

Evonik Industries baut derzeit in Marl eine Großanlage für funktionalisierte Polybutadiene, die mit einer Jahreskapazität von mehreren tausend Tonnen bereits Mitte dieses Jahres in Betrieb gehen soll.

$D^{a}$ as Spezialchemieunternehmen investiert hierfür an seinem weltweit größten Standort einen mittleren zweistelligen Millionen-€-Betrag und schafft 15 neue Arbeitsplätze. Funktionalisierte Polybutadiene, die Evonik unter dem Markennamen Polyvest HT vermarkten will, werden hauptsächlich in Dichtmassen für Isolierglasfenster sowie für Klebstoffe, beispielsweise in der Automobil- und Elektronikindustrie, verwendet.

Die neue Anlage kann im Chemiepark Marl die vorhandene Infrastruktur und Rohstoffversorgung sowie Synergien zu bestehenden Polybutadienanlagen opti- mal nutzen. Dazu Klaus Engel, Vorstandsvorsitzender von Evonik Industries: „Als eines der weltweit größten Spezialchemieunternehmen stehen wir zum Industriestandort Deutschland und sichern mit nachhaltigen Investitionen unsere industrielle Basis hierzulande ab." Und Ulrich Küsthardt, Leiter des Geschäftsbereichs Coatings \& Additives, ist davon überzeugt, dass der Bedarf nach Produkten, die dazu beitragen, Ressourcen effizienter zu nutzen, groß ist. Insbesondere in Deutschland und Europa sei der Markt für funktionalisierte Polybutadiene durch eine hohe Wachstumsdynamik geprägt. Sie werden beispielsweise bei Doppel- und Dreifachverglasung im Fensterbau sowie in Klebstoffen für die Leichtbauweise von Fahrzeugen eingesetzt. Im Automobilbau finden zunehmend Klebstoffe Einsatz, die als Ergänzung zu traditionellen Schweißverfahren bzw. als Strukturklebstoffe für nicht schweißbare Compositematerialien verwendet werden.

Mit der neuen Anlage erweitert Evonik das bestehende Leistungsportfolio aus Polyestern, Polymethacrylaten, amorphen Poly-alpha-Olefinen und Polybutadienen für die Kleb- und Dichtstoffindustrie.

\section{SKZ erweitert seine Klebaktivitäten}

D as Kunststoff-Zentrum SKZ in Würzburg erweitert seine maschinelle Ausstattung im Bereich der Klebtechnik durch eine Niederdruckplasma (ND)Plasmaanlage sowie einen $\mathrm{CO}_{2}$-Laser. Hierdurch können z. B. bei kundenspezifischen Anfragen neben den verschiedenen bereits vorhandenen Oberflächenaktivierungsmethoden für Kunststoffe (wie z.B. Atmosphärendruckplasma, Corona, Beflammung, usw.) auch Vorbehandlungen mit Niederdruckplasma (ND-Plasma) durchgeführt werden. Die neue ND-Plasma-Anlage „Pico“ der Firma Diener electronic bietet dabei neben der Aktivierung von Oberflächen für Klebtechnik diverse Möglichkeiten zur Reinigung sowie Beschichtung von Oberflächen - entsprechend durch Ätzund Polymerisationsprozesse.

Der $\mathrm{CO}_{2}$-Laser Speedy 100 der Trotec Laser $\mathrm{GmbH}$ mit einer Leistung von 45 Watt kann für diverse Fragestellungen wie z.B. Vergrößerung der spezifi- schen Oberfläche eines Kunststoff-Substrats durch partielle Ablation sowie Aufschmelzen, präzises Schneiden von Klebebändern, Folien sowie Platten, Markierungsaufgaben und Freilegung von Fasern bei Faserverbundwerkstoffen eingesetzt werden.

Der Einsatz dieser beiden Oberflächenvorbehandlungsanlagen in öffentlichen Forschungs- und Entwicklungsprojekten sowie zahlreichen industriellen Entwicklungsaufträgen komplettiert die Angebotspalette der am häufigsten eingesetzten physikalischen Oberflächenvorbehandlungsverfahren in der Kunststoffindustrie.

Das Kunststoff-Zentrum bietet außerdem zahlreiche zerstörenden und zerstörungsfreien Prüfmöglichkeiten sowie diverse Lösungen zum thermischen und klebtechnischen Fügen von polymeren Werkstoffen an.

Weitere Infos: www.skz.de

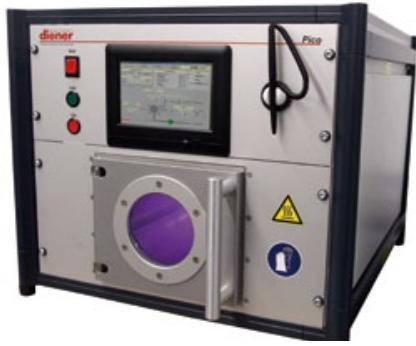

Niederdruckplasmaanlage für die Durchführung von Reinigungs-, Aktivierungs-, Ätz- sowie Poly-merisationsprozessen.

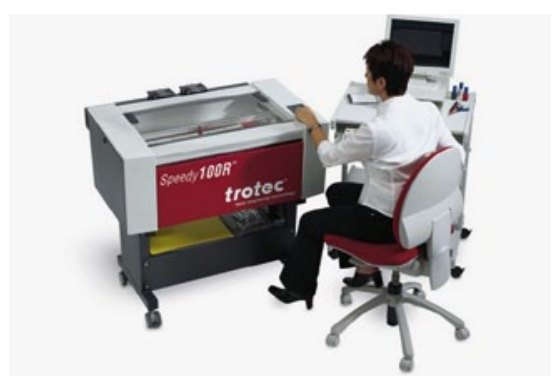

Dieser $\mathrm{CO}_{2}$-Laser kann z.B. zum Vergrößern der spezifischen Oberfläche eines KunststoffSubstrats, zum präzisen Schneiden von Klebebändern, für Markierungsaufgaben oder zum Freilegen von Fasern bei Faserverbundwerkstoffen eingesetzt werden.. 